SLAC-PUB-95-7010

Stanford ITP 95-22

hep-ph/9510220

\title{
Dynamical Relaxation of the Supersymmetric CP Violating Phases
}

\author{
Savas Dimopoulos \\ Theoretical Physics Division, CERN \\ CH-1211 Geneva 23, Switzerland \\ and \\ Physics Department \\ Stanford University \\ Stanford, CA 94305 \\ Scott Thomasit \\ Stanford Linear Accelerator Center \\ Stanford University \\ Stanford, CA 94309
}

A supersymmetric generalization of the Peccei-Quinn mechanism is proposed in which two $\mathrm{U}(1) \mathrm{CP}$ violating phases of the supersymmetric standard model are promoted to dynamical variables. This amounts to postulating the existence of spontaneously broken global symmetries in the supersymmetry breaking sector. The vacuum can then relax near a $\mathrm{CP}$ conserving point. As a consequence the strong $\mathrm{CP}$ and supersymmetric $\mathrm{CP}$ problems may be solved by similar mechanisms.

\footnotetext{
${ }^{\dagger}$ Work supported by the Department of Energy under contract DE-AC03-76SF00515.
} 


\section{Introduction}

The supersymmetric CP problem has emerged as one of the naturalness problems for the minimal supersymmetric standard model (MSSM). The present bounds on the electric dipole moments of atoms [1], molecules [2], and the neutron [3], restrict the the $\mathrm{CP}$ violating phases discussed below to be less than $\mathcal{O}\left(10^{-2}-10^{-3}\right)$ over much of the MSSM parameter space [4, 5]. A number of possible "solutions" to this problem have been suggested. In supergravity mediated supersymmetry breaking scenarios, if the MSSM remains the effective theory up to the Planck scale, it might be that the SUSY CP phases vanish at the Planck scale. The phases induced from the KobayashiMaskawa phase under renormalization group evolution are then sufficiently small at low energy [6]. However, in GUT theories much larger phases are induced from running between the Planck and GUT scales since the left and right handed quarks and leptons generally fall within the same multiplets [7]. Alternatively, if the superpartner masses are $\mathcal{O}(\mathrm{TeV})$ the bound on the phases is largely eliminated [8]. Finally, in scenarios with low energy gauge mediated SUSY breaking, the SUSY phases simply do not arise in many classes of models [9].

Here we point out that promoting the phases to dynamical variables can naturally lead to relaxation of the vacuum to (or near) a CP conserving point. This is an extension of the recent suggestion that the SUSY-flavor problem may be reduced by promoting flavor rotations among squarks to dynamical variables [10]. In this context it can be seen as a direct extension of the Peccei-Quinn mechanism for relaxation of the QCD vacuum angle [11, 12, 13]. In fact, DFSZ type axion models [13] can naturally arise if there is a spontaneously broken global $U(1)$ symmetry in the SUSY breaking sector. The existence of additional global $U(1)$ symmetries in the SUSY breaking sector can naturally lead to the relaxation mechanism proposed here for the SUSY CP phases. 


\section{CP Violating Phases in the MSSM}

Before discussing the relaxation mechanism, let us first review the origin of the CP violating phases in the MSSM. Since we are mainly interested in the flavor conserving phases we will work under the universality anzatz. With universality, four additional phases appear beyond the Kobayashi-Maskawa phase and QCD vacuum angle of the standard model. The first arises in the superpotential Higgs Dirac mass parameter $\mu$,

$$
W=\mu H_{u} H_{d}
$$

The remaining phases arise in the coefficients of the soft SUSY breaking parameters $m_{\lambda}, A$, and $m_{12}^{2}$,

$$
\mathcal{L}=-\frac{1}{2} m_{\lambda} \lambda \lambda-A\left(h_{u} Q H_{u} \bar{u}-h_{d} Q H_{d} \bar{d}-h_{e} L H_{d} \bar{e}\right)-m_{12}^{2} H_{u} H_{d}+\text { h.c. }
$$

where $\lambda$ are the gauginos, and $h_{i}$ the Yukawa couplings. Only two of the four phases are physical CP violating phases [14]. This is most readily seen by noticing that in the absence of non-gauge interactions there are two additional flavor conserving global $U(1)$ symmetries in the MSSM, a Peccei-Quinn and $R$ Peccei-Quinn symmetry [15]. Selection rules for the symmetries may therefore be used if the dimensionful parameters in the couplings given above which break the symmetries are treated as spurions with charges assigned to compensate those of the fields, as given in table 1. The selection rules limit the combinations of dimensionful parameters that can appear in a physical amplitude. Treating the dimensionful parameters as insertions, these are

$$
m_{\lambda} \mu\left(m_{12}^{2}\right)^{*}, \quad A \mu\left(m_{12}^{2}\right)^{*}, \quad A^{*} m_{\lambda}
$$

Among these there are two linearly independent phases which may be taken to be $\operatorname{Arg}\left(A \mu\left(m_{12}^{2}\right)^{*}\right)$ and $\operatorname{Arg}\left(A^{*} m_{\lambda}\right)$. 


\begin{tabular}{|c|c|c|}
\hline & $U(1)_{P Q}$ & $U(1)_{R-P Q}$ \\
\hline$m_{\lambda}$ & 0 & -2 \\
\hline$A$ & 0 & -2 \\
\hline$m_{12}^{2}$ & -2 & 0 \\
\hline$\mu$ & -2 & 2 \\
\hline$H_{u}$ & 1 & 0 \\
\hline$H_{d}$ & 1 & 0 \\
\hline$Q \bar{u}$ & -1 & 2 \\
\hline$Q \bar{d}$ & -1 & 2 \\
\hline$L \bar{e}$ & -1 & 2 \\
\hline
\end{tabular}

Table 1: Peccei-Quinn and $R$ charges of spurions and fields. 


\section{Relaxation of the Phases}

In order to motivate the relaxation solution to the SUSY CP problem it is instructive to review the role of nonlinearly realized global symmetries in this context. The non-supersymmetric standard model has, at the renormalizable level, two accidental global symmetries, namely baryon and lepton number. If these symmetries are realized nonlinearly there are Goldstone bosons which couple to the associated currents. However, if the scale of spontaneous symmetry breaking is large enough, the Goldstone bosons decouple and there are no effects at low energy [16]. The two Higgs doublet model has, in the absence of an $m_{12}^{2} H_{u} H_{d}$ term, an additional global Peccei-Quinn symmetry at the classical level [11]. This symmetry has a quantum mechanical anomaly with respect to QCD, so $\bar{\theta}_{Q C D}$ shifts under a Peccei-Quinn transformation. If this symmetry is realized in the Goldstone mode, i.e. $\operatorname{Arg}\left(m_{12}^{2}\right)$ is a dynamical variable, the associated pseudo-Goldstone boson (the axion) receives a potential from the explicit breaking due to the anomaly. It is technically natural that this potential is an extremum at points of enhanced symmetry. This is because if the symmetry is realized (nonlinearly or otherwise) in the relevant degrees of freedom, then the lowest order term in the potential near a symmetry point is bilinear in the fields. If such points are in fact minima, then since $\bar{\theta}_{Q C D} \rightarrow-\bar{\theta}_{Q C D}$ under $\mathrm{CP}$, the axion can relax to a $\mathrm{CP}$ conserving point, $\bar{\theta}_{Q C D}=0$ or $\pi$. The explicit breaking from the anomaly comes from a marginal operator, the topological charge density. Since QCD is asymptotically free, the low energy long distance dynamics can in principle determine the potential for the axion (as is usually implicitly assumed). Here low energy refers to the standard model particle content with renormalizable interactions. It is important to note that there can be additional explicit breakings from high energy short distance physics, which may disturb the alignment. High energy refers to for example GUT or Planck scale physics, which may contain additional degrees of freedom which do not conserve CP 
with respect to the low energy theory. However, in order for the mechanism to work, one must assume the Peccei-Quinn symmetry is respected by the high energy physics to a sufficiently high order in irrelevant operators [17].

The solution of the SUSY-CP problem we propose is to promote the phases appearing in (1) and (2) to dynamical variables. The soft terms of course arise from couplings with the SUSY breaking sector. Since the $\mu$ term must be of order the weak scale, the only reasonable assumption is that it too arises from a coupling to the SUSY breaking sector. Promoting the phases to dynamical variables therefore amounts to postulating the existence of spontaneously broken global symmetries in the SUSY breaking sector. The phases in (臬) and (2) are then the Goldstone bosons of these nonlinearly realized symmetries. In general all the phases need not be dynamical. However, for now we make the "maximal" assumption that all four phases are dynamical and investigate the consequences. As we show below, one phase is analogous to baryon and lepton number in that it is respected at the renormalizable level by couplings to the visible sector. The Goldstone boson which could be associated with this symmetry therefore has no effect at low energy if the SUSY breaking scale is large enough. A second symmetry is analogous to the Peccei-Quinn symmetry in the two Higgs doublet model, and can lead to the Peccei-Quinn mechanism for the relaxation of $\bar{\theta}_{Q C D}$ [18 (if respected to high enough order). The remaining two phases are the physical SUSY CP violating phases. As we show below any symmetries associated with these turn out to be explicitly broken by couplings with the visible sector. Integrating out the visible sector then produces a potential with minima near $\mathrm{CP}$ conserving points for the phases. This leads to a relaxation mechanism analogous to the Peccei-Quinn mechanism.

The relaxation mechanism requires that the vacuum energy be a minimum at (or near) a CP conserving point for the phases (3). The global symmetries associated with the phases must therefore be explicitly broken by some couplings. The possible terms appearing in the vacuum energy which 
depend on the phases are limited by the selection rules given above. Treating the dimensionful parameters as background spurions and including the Higgs bosons, which acquire an expectation value at low energy, the possible phase dependent terms are

$$
\begin{array}{cc}
m_{\lambda} \mu H_{u} H_{d} & m_{\lambda} \mu\left(m_{12}^{2}\right)^{*} \\
A \mu H_{u} H_{d} & A \mu\left(m_{12}^{2}\right)^{*} \\
A^{*} m_{\lambda} H_{u}^{*} H_{u} & A^{*} m_{\lambda} H_{d}^{*} H_{d} \\
A^{*} m_{\lambda} \Lambda^{2} &
\end{array}
$$

where each term appears with + h.c., and $\Lambda$ is a dimensionful scale discussed below. In general there are contributions to the terms above from both long and short distance physics. The long distance contributions from light degrees of freedom come from diagrams such as those given in Figs. 1-4. Just on dimensional grounds all the terms in (4), except the last one, correspond to marginal operators. For the marginal operators, at worst, all logarithmic energy scales contribute equally to the coefficients. The contributions from integrating out light degrees of freedom can therefore dominate the short distance contributions by $\mathcal{O}\left(\ln \left(\Lambda^{2} / m_{W}^{2}\right)\right)$, where $m_{W}$ is the weak scale and $\Lambda$ is the scale at which SUSY breaking is transmitted to the visible sector ( $\Lambda \sim M_{p}$ in hidden sector models). In this case the breaking of the global symmetries can come mainly from the visible sector, and need not be particularly sensitive to short distance physics. This relative insensitivity to short distance physics is in contrast to the situation for dynamical squark flavor matrices [10], dynamical Yukawa couplings [19, 20], or a dynamical determination of the SUSY breaking scale in no-scale type models [21, 22]; in these cases the potential is quadratically sensitive to the short distance physics [23]. Likewise here, the last term in (4) corresponds to a relevant operator. Integrating out light degrees of freedom therefore gives (in the absence of a regulator) a quadratically divergent contribution to the operator, 
proportional to $\Lambda^{2}$. An example of such a three loop diagram is obtained from Fig. 3. with $H_{u}$ contracted with $H_{u}^{*}$. Because of the quadratic divergence, this contribution to the vacuum energy is very sensitive to the short distance physics. This sensitivity implies the precise description of the short distance contribution is in fact scheme dependent. For example, in dimensional regularization there are no quadratic divergences, and the $\Lambda^{2}$ piece comes from the matching conditions at the scale $\Lambda$. The potential for the phase $\operatorname{Arg}\left(A^{*} m_{\lambda}\right)$ is therefore essentially determined by physics at the scale $\Lambda$.

Now consider the form of the potential arising from (田) for the Goldstone bosons associated to the $\mathrm{CP}$ violating phases in (3). First note that in the ground state the $m_{12}^{2} H_{u} H_{d}$ term in the potential fixes $\operatorname{Arg}\left(H_{u} H_{d}\right)=$ $-\operatorname{Arg}\left(m_{12}^{2}\right)$. Ignoring for the moment the Kobayashi-Maskawa phase and any flavor changing phases, the long distance contributions of the types given in Figs. 1-4 to the marginal operators then all go like

$$
\sum_{i} c_{i} m_{W}^{4} \cos \left(\phi_{\alpha}+\delta_{i}\right)
$$

where $c_{i}$ is the magnitude of the $i$ th diagram, and $\phi_{\alpha}=\operatorname{Arg}\left(m_{\lambda} \mu\left(m_{12}^{2}\right)^{*}\right)$ or $\operatorname{Arg}\left(A \mu\left(m_{12}^{2}\right)^{*}\right)$. Since the only $\mathrm{CP}$ violating phase in the lowest order diagrams is the phase $\phi_{\alpha}$ itself, $\delta_{i}=0$ or $\pi$. The lowest order long distance contributions to the vacuum energy therefore necessarily have minima at $\mathrm{CP}$ conserving points. For the relevant operator the lowest order contributions go like

$$
\sum_{i} c_{i} m_{W}^{2} \Lambda^{2} \cos \left(\phi+\delta_{i}\right)
$$

where $\phi=\operatorname{Arg}\left(A^{*} m_{\lambda}\right)$. Again, the long distance contributions, such as that in Fig. 3, give $\delta_{i}=0$ or $\pi$.

The short distance pieces, however, in general have arbitrary $\delta$. In order to proceed without simply assuming a tuning of the short distance phases we must therefore assume that the global symmetry associated with $\operatorname{Arg}\left(A^{*} m_{\lambda}\right)$ 
is realized in the short distance physics at the scale $\Lambda$. In addition we must assume that the short distance physics has the same definition of CP as the long distance physics, so that the potential has extrema at the CP conserving points, $\delta=0$ or $\pi$ [24]. This could occur for example if CP is a symmetry of the full theory, and only broken spontaneously below the scale $\Lambda$. In string theory, where CP is a symmetry [25], with hidden sector SUSY breaking this could occur if the scale of spontaneous $\mathrm{CP}$ violation is between the Planck and intermediate SUSY breaking scale $M_{I} \sim \sqrt{m_{W} M_{p}}$. Note that without the relaxation mechanism the soft parameters and $\mu$ would not in general be real in this case. With these assumptions about the short distance physics, since the combinations of phases that appear in the vacuum energy are precisely those that appear in any CP odd observable, there is a ground state in which all physical amplitudes (proportional to the terms in (3)) are CP conserving. This can also be seen by starting from the original basis for the phases in (1) and (2). $U(1)_{P Q}$ and $U(1)_{R-P Q}$ redefinitions may always be used to transform to a basis in which any two of the phases vanish, for example $\phi_{A}=\phi_{m_{12}^{2}}=0$. In the $\mathrm{CP}$ conserving ground state the alignment of $\operatorname{Arg}\left(A^{*} m_{\lambda}\right)$ then forces $\phi_{m_{\lambda}}=0$ or $\pi$, and the alignment of $\operatorname{Arg}\left(A \mu\left(m_{12}^{2}\right)^{*}\right)$ forces $\phi_{\mu}=0$ or $\pi$.

The alignment of the phases described above can be disturbed in a number of ways. Higher order loop diagrams of light degrees of freedom can be proportional to products of the invariants (4), and therefore have potentials proportional to $\cos \left(n \phi_{\alpha} \pm n^{\prime} \phi_{\beta}+\delta\right)$. These however are suppressed by at least two additional loop factors and do not shift the CP conserving minima. The Kobayashi-Maskawa phase can in principle shift the minimum of the potential from a CP conserving point. To form the Jarlskog invariant $J=\operatorname{Im}\left(V_{u d} V_{t d}^{*} V_{t b} V_{u b}^{*}\right)$ however requires at least four $S U(2)$ gauge couplings. This requires at least two additional loops compared with the lowest order diagrams, and is therefore down by at least $\mathcal{O}\left((\alpha / 4 \pi)^{2} J\right)$. GIM suppression among the squarks would reduce this contribution even further. The 
Kobayashi-Maskawa phase therefore does not significantly shift the minima from CP conserving points. The alignment can also be disturbed by explicit breaking by the short distance physics of both CP symmetry and the global symmetries in the hidden sector which make the phases dynamical. Here, just as for the Peccei-Quinn mechanism, we must assume that the minimum is not shifted to high enough order in irrelevant operators. However, since the bound on the phases is $\mathcal{O}\left(10^{-2}-10^{-3}\right)$ this is not nearly as restrictive as for the axion.

\section{The Physical (Pseudo)-Goldstone Bosons}

The physical pseudo-Goldstone bosons are related to the phases by $\phi_{\alpha}=$ $G_{\alpha} / f_{\alpha}$. The decay constants $f_{\alpha}$ are essentially the expectation values for fields in the SUSY breaking sector which transform under the symmetries. All the Goldstone bosons couple to the visible sector through dimensionful couplings with a suppression of $1 / f$,

$$
\begin{gathered}
W=|\mu| e^{i Q_{\mu \alpha} \phi_{\alpha} / f_{\alpha}} H_{u} H_{d} \\
\mathcal{L}=-\frac{1}{2}\left|m_{\lambda}\right| e^{i Q_{\lambda \alpha} \phi_{\alpha} / f_{\alpha}} \lambda \lambda-|A| e^{i Q_{A \alpha} \phi_{\alpha} / f_{\alpha}}\left(h_{u} Q H_{u} \bar{u}-h_{d} Q H_{d} \bar{d}-h_{e} L H_{d} \bar{e}\right) \\
-\left|m_{12}^{2}\right| e^{i Q_{u d \alpha} \phi_{\alpha} / f_{\alpha}} H_{u} H_{d}+\text { h.c. }
\end{gathered}
$$

where the $Q_{i \alpha}$ depend on the global charge assignments in the SUSY breaking sector. For $f$ above the weak scale the Goldstone bosons are essentially "invisible" to laboratory experiments. The masses for the Goldstone bosons corresponding to SUSY CP violating phases depend on the magnitude of (4) and the decay constant. The mass for the linear combination lifted by the marginal operators is then $m_{G} \sim(\sqrt{\alpha / 4 \pi}) m_{W}^{2} / f$, where $\alpha / 4 \pi$ counts the loop factor, while the mass for the combination lifted by the relevant operator is $m_{G} \sim m_{W} \Lambda / f$. In the universal case with four dynamical phases, due to the $U(1)_{P Q}$ and $U(1)_{R-P Q}$ symmetries of the $\mu$ and soft terms, the two 
linear combinations of phases orthogonal to the physical SUSY CP violating phases do not receive a perturbative potential from coupling with the visible sector (田). One linear combination is anomaly free with respect to QCD, and so receives a potential only from any explicit breaking from short distance physics. The other linear combination is anomalous and receives a potential from the QCD topological charge density. If this symmetry is respected by the short distance physics to high enough order in irrelevant operators, the Goldstone boson just acts as an invisible axion with mass $m \sim m_{\pi} f_{\pi} / f$, thereby solving the strong $\mathrm{CP}$ problem. The Peccei-Quinn mechanism can therefore be wedded with the proposal to solve the SUSY CP problem by postulating global symmetries in the SUSY breaking sector.

Depending on the mechanism which transmits SUSY breaking to the visible sector, $f$ could be anywhere between just above the weak scale to the Planck scale. One possibility is a renormalizable hidden sector in which SUSY is broken in the flat space limit, but transmitted to the visible sector by gravitational strength interactions. The scalar expectation values in the hidden sector are then of order the SUSY breaking scale, $f \sim M_{I} \sim \sqrt{m_{W} M_{p}}$. If, as suggested above, both the axion and the pseudo-Goldstone bosons responsible for relaxing the SUSY CP phases all arise from spontaneously broken global symmetries in the SUSY breaking sector, then apparently this type of hidden sector naturally gives a decay constant in the "axion window" allowed by astrophysical and cosmological bounds on the axion [26]. From (8) it is apparent that this axion couples both as a hadronic axion [12] through the gluino mass term and as a DFSZ axion [13 through the scalar Higgs term. Note that the small coupling introduced in the original DFSZ models by hand appears here automatically as $m_{W} / f$. With this type of hidden sector the pseudo-Goldstone bosons associated with the SUSY CP violating phases do not lead to excessive cooling of astrophysical systems, and are heavier than the axion and therefore not overproduced in the early universe.

With a renormalizable hidden sector it turns out that $A$ terms arise only 
from Kahler potential couplings and are always real [27]. In this case there are only three possible dynamical phases in the universal case, thereby eliminating the (potentially massless) anomaly free linear combination. One may be tempted to identify one of the required Goldstone bosons with the $R$ axion which plays a role in all known models of dynamical SUSY breaking based on a nonperturbative superpotential. However, for a renormalizable hidden sector, cancelation of the cosmological constant by adjustment of the superpotential, explicitly breaks the $R$ symmetry 28].

It is also worth noting that in renormalizable hidden sector models the heavier pseudo-Goldstone boson has a sizeable mass, $m \sim M_{I}$. This is because of the sensitivity of the relevant operator to physics at the scale $\Lambda \sim M_{p}$. The mass in other types of SUSY breaking sectors is parametrically less than the intrinsic SUSY breaking scale.

Another possibility is a nonrenormalizable hidden sector in which SUSY is restored in the flat space limit. In this case the fields can have $f \sim M_{p}$. With a nonrenormalizable hidden sector it is possible to cancel the cosmological constant by adjusting the Kahler potential without explicitly violating $R$ symmetry. It it is then possible in principle to identify the $R$ axion with one of the Goldstone bosons. If both the axion and pseudo-Goldstone bosons arise from spontaneously broken global symmetries in this type of hidden sector, they will generally be overproduced in the early universe. They may be diluted by a period of very late inflation however [29].

One interesting consequence of the relaxation mechanism for the SUSY $\mathrm{CP}$ phases is the possibility of a long range force. If the minimum of the potential does not align precisely with a $\mathrm{CP}$ conserving point, $\phi_{0} \neq 0$ or $\pi$, the interactions (8) lead to scalar couplings of the pseudo-Goldstone bosons to matter proportional to mass. This gives rise to a coherent potential between macroscopic bodies

$$
V \simeq-g^{2} \phi_{0}^{2} \frac{m_{i} m_{j}}{4 \pi f^{2}} \frac{e^{-m r}}{r}
$$


where $g \sim \mathcal{O}(1)$ is a dimensionless coupling which depends on the charges appearing in $(\mathbb{B}), \phi_{0}$ is the minimum of the phase potential $\bmod \pi$, and $m^{-1}$ is the Compton wavelength. The (lighter) Goldstone boson, which receives a mass from the marginal operators, gives the longest range force. For $f \sim M_{p}$ the Compton wavelength is $\mathcal{O}\left(10^{-1}-100\right) \mathrm{cm}$, and is weaker than gravity by roughly the factor $\phi_{0}^{2}$. As argued above, the shift in the potential from long distance physics can be quite small in the universal case, but short distance physics or non-universality can in principle disturb the alignment. Notice that for $\phi_{0} \neq 0$ the magnitude of CP odd observables, such as electric dipole moments, is correlated with the strength of the long range force. This may be the best laboratory signal for the relaxation mechanism.

\section{Conclusions}

Promoting the SUSY CP phases to dynamical variables allows the possibility that the vacuum can relax to a ground state at or near a CP conserving point for these phases. The long distance contribution to the potential for the flavor conserving phases can in fact have a minimum near a CP conserving point. Although not calculable in the low energy theory, it is technically natural that the short distance potential also lies near a CP conserving point. Promoting the phases to dynamical variables amounts to postulating the existence of spontaneously broken global symmetries in the SUSY breaking sector. This requires protecting (in the limit of decoupling the visible sector) some compact flat directions in the SUSY breaking sector, which is of course possible with symmetries. This is in contrast to some other mechanisms for dynamically determining low energy parameters which require protecting noncompact flat directions in the presence of SUSY breaking. Noncompact symmetries can do this at the classical level, but are generally violated quantum mechanically. Also note that since the relaxation of these phases requires global symmetries in the SUSY breaking sector, other CP violating phases 
unrelated to this sector, such as the Kobayashi-Maskawa phase, of course need not be dynamical. Finally, the mechanism for the relaxation of the SUSY CP violating phases is a generalization of the Peccei-Quinn mechanism for the solution of the strong CP problem.

After this work was completed it was brought to our attention that Ref. [30] considered the possibility of dynamical phases in models of moduli dominated supersymmetry breaking.

\section{References}

[1] S. Murthy, D. Krause, Z. Li, and L. Hunter, Phys. Rev. Lett. 63 (1989) 965; E. Oteiza, R. Hoare, T. Chupp, Bull. Am. Phys. Soc. 37 (1992) 947; J. Jacobs, W. Klipstein, S. Lamoreaux, B. Heckel, and E. N. Fortson, Phys. Rev. Lett. 71 (1993) 3782; E. Cummins, S. Ross, D. Demille, and C. Regan, Phys. Rev. A 50 (1994) 2960.

[2] D. Cho, K. Stangster, and E. Hinds, Phys. Rev. Lett. 63 (1989) 2559; Phys. Rev. A 44 (1991) 2783.

[3] K. Smith et al., Phys. Lett. B 234 (1990) 191; I. Altarev et al., Phys. Lett. B 276 (1992) 242.

[4] J. Ellis, S. Ferrara, and D. Nanopoulos, Phys. Lett. B 114 (1982); W. Buchmuller and D. Wyler, Phys. Lett. B 121 (1983) 321; J. Polchinski and M. Wise, Phys. Lett. B 125 (1983) 393.

[5] W. Fischler, S. Paban, and S. Thomas, Phys. Lett. B 289 (1992) 373; S. Barr, Int. J. Mod. Phys. A 8 (1993) 209.

[6] S. Bertolini and F. Vissani, Phys. Lett. B 324 (1994) 164; T. Inui, Y. Mimura, N. Sakai, and T. Sasaki, Nucl. Phys. B 449 (1995) 49. 
[7] S. Dimopoulos and L. Hall, Phys. Lett. B 344 (1995) 185; R. Barbieri, L. Hall, and A. Strumia, Nucl. Phys. B 445 (1995) 219.

[8] Y. Kizukuri and N. Oshimo, Phys. Rev. D 45 (1992) 1806; Phys. Rev. D 46 (1992) 3025.

[9] M. Dine and A. Nelson, Phys. Rev. D 48 (1993) 1277; M. Dine, A. Nelson, and Y. Shirman, Phys. Rev. D 51 (1994) 1362.

[10] S. Dimopoulos, G. Guidice, and N. Tetradis, preprint CERN-TH/95-90, hep-ph/9504296.

[11] R. Peccei and H. Quinn, Phys. Rev. Lett. 38 (1977) 1440.

[12] J. Kim, Phys. Rev. Lett. 43 (1979) 103.

[13] A. Zhitnitskii, Yad. Fiz. 31 (1980) 497 [Sov. J. Nucl. Phys. 31 (1980) 260]; M. Dine, W. Fischler, and M. Srednicki, Phys. Lett. B 109 (1981) 199.

[14] M. Dugan, B. Grinstein, and L. Hall, Nucl. Phys. B 255 (1985) 413.

[15] Under an $R$ transformation the scalar, fermionic, and auxillary components of a chiral superfield transform as $\phi \rightarrow e^{i \alpha R} \phi, \psi \rightarrow e^{i \alpha(R-1)} \psi$, and $F \rightarrow e^{i \alpha(R-2)} F$ respectively, where $R$ is the $R$ charge. The superpotential has $R$ charge $R(W)=2$, and a gauge superfield has $R$ charge $R\left(W^{\alpha}\right)=1$.

[16] Y. Chikashige, R. Mohapatra, and R. Peccei, Phys. Lett. B 98 (1981) 265; W. Fischler, G. Guidice, R. Leigh, S. Paban, and S. Thomas, Phys. Lett. B 266 (1991) 408.

[17] H. Georgi, L. Hall, and M. Wise, Nucl. Phys. B 192 (1981) 409; M. Dine and N. Seiberg, Nucl. Phys. B 273 (1986) 109; M. Kamionkowski and 
J. March-Russell, Phys. Lett. B 282 (1992) 137; R. Holman, S. Hsu, T. Kephart, E. Kolb, R. Watkins, and L. Widrow, Phys. Lett. B 282 (1992) 132; S. Barr and D. Seckel, Phys. Rev. D 46 (1992) 539.

[18] J. Ellis, K. Enqvist, and D. Nanopoulos, Phys. Lett. B 151357.

[19] C. Kounnas, F. Zwirner, and I. Pavel, Phys. Lett. B 335 (1994) 403; P. Binetruy and E. Dudas, Phys. Lett. B 338 (1994) 403; C. Kounnas, I. Pavel, G. Ridolfi, and F. Zwirner, preprint CERN-TH/95-11, hepph/9502318.

[20] Y. Nambu, preprint EFI 92-37; V. Zakharov, in "Properties of SUSY particles" (L. Difarelli and V. Khoze eds., World Scientific, Singapore, 1993) p.65.

[21] E. Cremmer, S. Ferrara, C. Kounnas, and D. Nanopoulos, Phys. Lett. B 133 (1983) 61; J. Ellis, A. Lahanas, D. Nanopoulos, and K. Tamvakis, Phys. Lett. B 134 (1984) 429; J. Ellis, C. Kounnas, and D. Nanopoulos, Nucl. Phys. B 241 (1984); Nucl. Phys. B 247 (1984) 373.

[22] S. Ferrara, C. Kounnas, and F. Zwirner, Nucl. Phys. B 429 (1994) 589; Nucl. Phys. B 433 (1995) 255.

[23] J. Bagger, E. Poppitz, and L. Randall, preprint EFI-95-21, hepph/9505244; N. Arkani-Hamed and H.-C. Cheng, unpublished.

[24] It is worth noting that the technically natural option of postulating a minimum of the short distance potential at an enhanced symmetry point can also be applied to the case of dynamical squark flavor rotations, but does not appear to be available for dynamical Yukawa couplings or the dynamical determination of the SUSY breaking scale in no-scale type models. 
[25] M. Dine, R. Leigh, and D. McIntire, Phys. Rev. Lett. 69 (1992) 2030; K. Choi, D. Kaplan, and A. Nelson, Nucl. Phys. B 391 (1993) 515.

[26] For a review of astrophysical and cosmological bounds on the axion and light Goldstone bosons see G. Raffelt, Phys. Rep. 198 (1990) 1.

[27] J. Feng, N. Polonsky, and S. Thomas, SLAC-PUB-95-7050.

[28] T. Banks, D. Kaplan, and A. Nelson, Phys. Rev. D 49 (1994) 779; J. Bagger, E. Poppitz, and L. Randall, Nucl. Phys. B 426 (1994) 3.

[29] S. Dimopoulos and L. Hall, Phys. Rev. Lett. 60 (1988) 1899.

[30] K. Choi, Phys. Rev. Lett. 72 (1994) 1592. 


\section{Figure Captions}

Figure 1. One loop contribution to the vacuum energy proportional to $m_{\lambda} \mu H_{u} H_{d}+$ h.c. Contracting $H_{u}$ and $H_{d}$ gives a two loop contribution proportional to $m_{\lambda} \mu\left(m_{12}^{2}\right)^{*}+$ h.c.

Figure 2. One loop contribution to the vacuum energy proportional to $A \mu H_{u} H_{d}+$ h.c. Contracting $H_{u}$ and $H_{d}$ gives a two loop contribution proportional to $A \mu\left(m_{12}^{2}\right)^{*}+$ h.c.

Figure 3. Two loop contribution to the vacuum energy proportional to $m_{\lambda} \mu H_{u} H_{d}+$ h.c. and $A^{*} m_{\lambda} H_{u}^{*} H_{u}+$ h.c. Contracting $H_{u}$ and $H_{u}^{*}$ gives a quadratically divergent contribution proportional to $A^{*} m_{\lambda}+$ h.c..

Figure 4. A three loop contribution to the vacuum energy proportional to $A \mu\left(m_{12}^{2}\right)^{*}+$ h.c. 


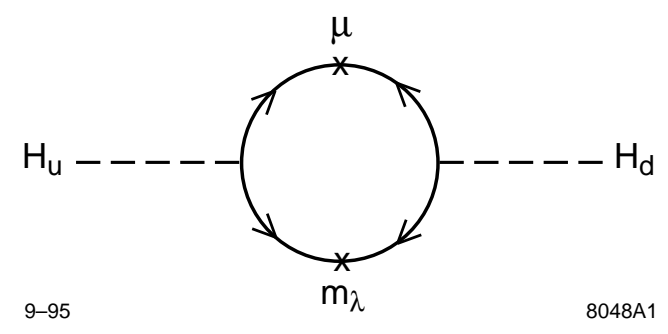




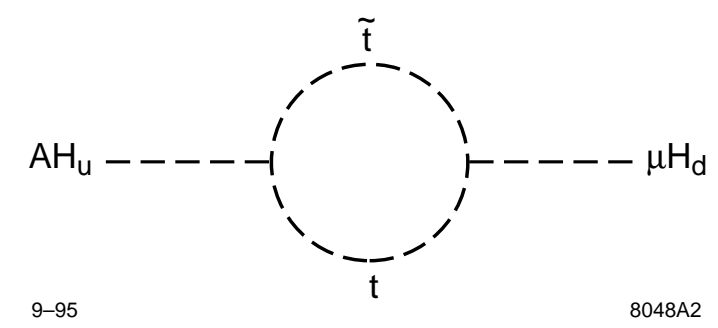




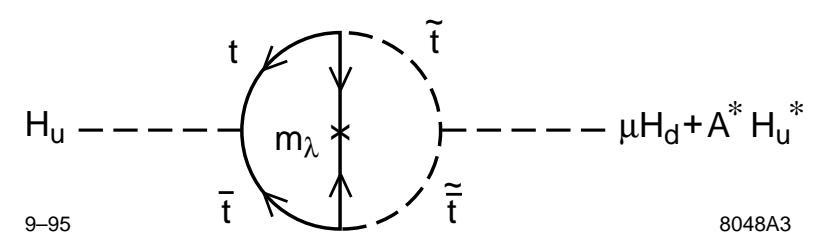




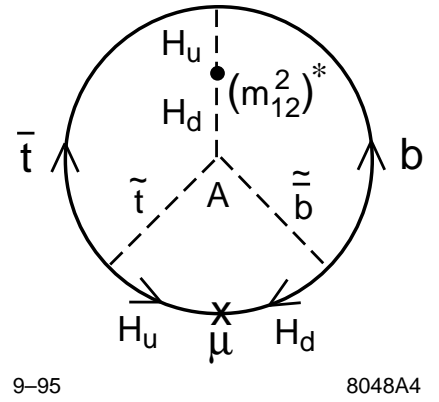

